Original Article

\title{
ROLE OF NITRIC OXIDE AND ARGINASE IN THE PATHOGENESIS OF ORAL CANCER
}

\author{
Nidarsh D. Hegde ${ }^{1}$, Suchetha Kumari ${ }^{2}$, Mithra N. Hegde ${ }^{3}$, Shilpa Shetty ${ }^{4}$ \\ ${ }^{1}$ Department of Oral and M axillofacial Surgery, A.B. Shetty M emorial Institute of Dental Sciences, Nitte University, \\ M angalore - 575 018, India. ${ }^{2}$ Department of Biochemistry, K. S. Hegde M edical Academy, Nitte University, M angalore - \\ 575 018, India. ${ }^{3}$ Department of Conservative Dentistry and Endodontics, A. B. Shetty M emorial Institute of Dental \\ Sciences, Mangalore - 575 018, India, \\ ${ }^{4}$ Central Research Laboratory, Nitte University, Mangalore - 575 018, India.
}

\author{
Correspondence: \\ Nidarsh D. Hegde, \\ E-mail: drhegdedental@gmail.com
}

\begin{abstract}
:
The aim of the present study is to evaluate the status of serum nitric oxide and arginase activity in oral cancerous condition. The study consisted of 80 individuals. The subjects were divided into normal individuals and individuals with oral cancer. Blood sample was collected from each subject and serum separated. Nitric oxide concentration was measured as total nitrates and nitrites by the Griess reaction method and arginase activity was estimated by diacetylmonoxime method.

The results of this study showed significantly increased levels of nitric oxide and arginase activity in oral cancerous condition when compared to normal individuals. From the study it can be concluded that during carcinogenesis and tumor progression, the level of nitric oxide and arginase activity increases. This could result from a generalized increased nitric oxide synthesis throughout the body of the cancer patient or reflect increased nitric oxide degradation promoted by oxidative stress.
\end{abstract}

Keywords: Nitric oxide, Arginase, Oral Cancer

\section{Introduction:}

Oral cancer is part of a group of cancers called head and neck cancer. Oral cancer can develop in any part of the oral cavity and oropharynx. Risk factors for oral cancer are tobacco, alcohol use, exposure to sunlight, chronic irritation, lack of fruits and vegetables in diet, alcohol containing mouth wash, human papilloma virus infection and immune system suppression ${ }^{1}$.

Nitric oxide (NO) plays multiple roles in both intracellular and extracellular signaling mechanisms. This highly reactive, yet simple molecule is produced in the body by the isoenzyme nitric oxide synthase (NOS) using L-arginine as a substrate. Three isoforms of NOS have been characterized. Two of them are constitutive NOS (CNOS) and the third isinducible (iNOS) by endotoxins and cytokines. ${ }^{2}$. Reaction of nitric oxide with oxygen or other free radicals generates reactive nitrogen species (RNS), which cause multiple biological effects ${ }^{3}$. Overexpression of NOS in chronic inflammation can lead to genotoxicity. Nitric oxide may mediate DNA damage through the formation of carcinogenic nitrosamines, generation of RNS and inhibition of DNA damage repair mechanism. It can thus be considered as a tumor initiating agent ${ }^{4}$. However, Nitric oxide may also have an impact on other stages of cancer development. These effects of nitric oxide are broad, with its involvement ranging from cellular transformation and formation of neoplastic lesions to the regulation of various other aspects of tumor biology $\mathrm{y}^{2 \cdot 6}$.

Inducible nitric oxide synthase is responsible for generating high levels of nitric oxide in tissues. Increased iNOS expression has been demonstrated in a number of carcinomas including head and neck squamous cell carcinoma ${ }^{7.9}$.Nitric oxide up regulates cyclo-oxygenase- 2 and synthesis of several other inflammatory cytokines. Inflammation and thrombotic complication are usually associated with malignancy. Threshold levels of nitric oxide required for the activation and stabilization of key proteins involved in carcinogenesis including p53, ERK, AKt and $\mathrm{HIF}^{10}$.

L-Arginine is the common substrate for two enzymes, arginase and nitric oxide synthase (NOS). Arginase converts 
L-arginine to L-ornithine, which is the precursor of polyamines, which are essential components of cell proliferation. Arginase modulates nitric oxide production in activated macrophages. In macrophages and many other cell types, L-arginine is used as substrate by both nitric oxide synthase and arginase to produce nitric oxide and urea, respectively ${ }^{11}$. Arginase reflects the type of inflammatory response in a specific disease process .The availability of arginine during critical illness may be regulated by arginase activity. Thus arginase expression appears to be essential in the regulation of the cellular immune response and the inflammatory process during critical illness ${ }^{12-14}$.

\section{Materials And Method:}

This study was conducted in the Central Research Laboratory of Nitte University after the approval from institutional ethical committee.

\section{Subjects:}

The study involved 80 patients reporting to the Department of oral medicine and Radiology, A.B Shetty M emorial Institute of Dental Sciences

\section{Study group:}

Includes total of 60 subjects with oral cancer were included.

\section{Control group:}

20 healthy adults without oral cancer were included.

\section{Inclusion criteria :}

G Patients between age group 20-60 years.

$\mathrm{G}$ Include clinically diagnosed cases of oral submucous fibrosis and histopathologically confirmed cases of leukoplakia and erythroplakia.

G Include clinically diagnosed and histopathologically confirmed cases of oral cancer.

\section{Exclusion criteria :}

G Subjects with other systemic diseases.

G Subjects taking any medications/antioxidant supplementation will not be included in the study.

A detailed case history of the patient was taken. A case history format was filled, with a informed consent which was duly signed by each patient.

\section{Collection of serum :}

$5 \mathrm{ml}$ of blood samples of the subjects was collected by vein puncture, centrifuged to separate the serum and stored at $4^{\circ} \mathrm{C}$.

Estimation of Nitric oxide $\left(\mathrm{NO}_{2}+\mathrm{NO}_{3}\right)$ concentration by Griess reaction method

Nitric oxide concentration was measured as total nitrates and nitrites $\left(\mathrm{NO}_{2}+\mathrm{NO}_{3}\right)$ by the Griess reaction method. Absorbance is read at $550 \mathrm{~nm}$. Concentration is determined using standard graph.

\section{Estimation of Arginase Activity by Diacetylmonoxime method}

Diacetylmonoxime reacts with urea formed by arginase action under strongly acidic conditions in the presence of ferric ion and thiosemicarbazide giving a pink coloured diacetyl complex which is read spectrophotometrically at $540 \mathrm{~nm}$. Concentration is determined using standard graph.

\section{Statistical analysis:}

Results are presented as mean \pm standard deviation value and statistically analyzed by Student ' $t$ ' test. A ' $p$ ' value of 0.05 or less was considered significant

\section{Result :}

The present study involves the estimation of the levels of nitric oxide and arginase activity in oral cancerous condition and their comparison between diseased and normal individuals. The results are expressed in Table-1 and Fig-1, 2 .

In this study nitric oxide level was found to be increased significantly ( $p=0.0025)$ in oral cancerous condition when compared to normal individuals. Similarly arginase activity was found to be increased significantly $(p=0.0130)$ in oral cancerous condition when compared to normal individuals.

\section{Discussion :}

Cancer is an undifferentiated mass of cell. Cancer occurs due to the failures of the mechanisms that usually control the growth and regulation of the cell. The losers of cellular regulation that give rise to most or all cases of cancer are due to genetic damage that is often accompanied with by influences of tumor promoting chemicals, hormones and 
some time viruses.

The role of nitric oxide (NO) is multidimensional. It functions as an intracellular messenger and is also implicated as a deleterious agent in various pathophysiological conditions including cancer, inflammatory conditions and autoimmune diseases. Chronic inflammation can lead to the production of NO, which in turn has the potential to mediate DNA damage directly, or indirectly through the generation of more persistent $\mathrm{RNS}^{15}$.

NOS activity has been detected in tumor cells of various histogenetic origins and has been associated with tumor grade, proliferation rate and expression of important signaling components associated with cancer development. NO pathway appears to play a key role in angiogenesis and spread in patients with head and neck cancer ${ }^{7}$. Increased iNOS expression and the generation of high NO levels might have role in oral squamous cell carcinoma development. Thus NO in cancer will have preformed therapeutic implications for the diagnosis and treatment of disease. The activation of iNOS or Arginase (or both) reflects the type of inflammatory response in a specific disease process ${ }^{8}$. Arginase converts the arginine to ornithine, which is nonprotein amino acid utilized by the tumor cells as nutrient for their growth ${ }^{16}$.

In this study nitric oxide level and arginase activity is found to be increased significantly in oral cancer patients when compared with healthy subjects. This shows that, elevated level of nitric oxide and arginase, are involved in carcinogenesis and tumor progression. Since, arginase regulates the nitric oxide production, the level of increase in arginase may be higher compared to increase in nitric oxide level.

This indicates that both nitric oxide and arginase play a role during carcinogenesis and tumor progression.

\section{Conclusion :}

Our study showed that serum nitric oxide level and arginase activity is higher in oral cancerous condition when compared to normal individuals. From the results obtained, it can be concluded that during carcinogenesis and tumor progression, the level of nitric oxide and arginase activity increases. This could result from a generalized increased nitric oxide synthesis throughout the body of the cancer patient or reflect increased nitric oxide degradation promoted by oxidative stress. Thus indicates that both nitric oxide and arginase play a role during carcinogenesis and tumor progression.

Table1: Comparison of serum nitric oxide levels and arginase activity in patients with oral cancer and normal individuals

\begin{tabular}{|l|c|c|c|}
\hline & $\begin{array}{c}\text { NORMAL } \\
\mathbf{N = 2 0}\end{array}$ & $\begin{array}{c}\text { ORAL CANCER } \\
\mathbf{N = 6 0}\end{array}$ & ' $\mathbf{p}$ ' VALUE \\
\hline NITRIC OXIDE & $40.57 \pm 11.82$ & $93.68 \pm 49.60$ & 0.0025 \\
\hline ARGINASE & $5.844 \pm 1.328$ & $19.3 \pm 10.02$ & 0.0130 \\
\hline
\end{tabular}

' $p$ ' $₫ .05$ is statistically significant. Statistical comparisons were performed by Student 't' test. Data are expressed as mean „SD.

Figure1: Comparison of serum nitric oxide levels in patients with oral cancer and normal individual

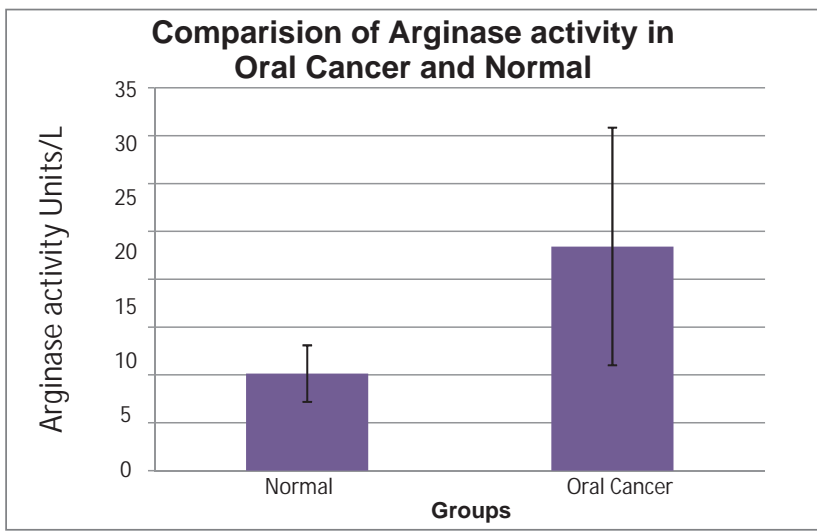

Figure 2: Comparison of serum arginase activity in patients with oral cancer and normal individuals

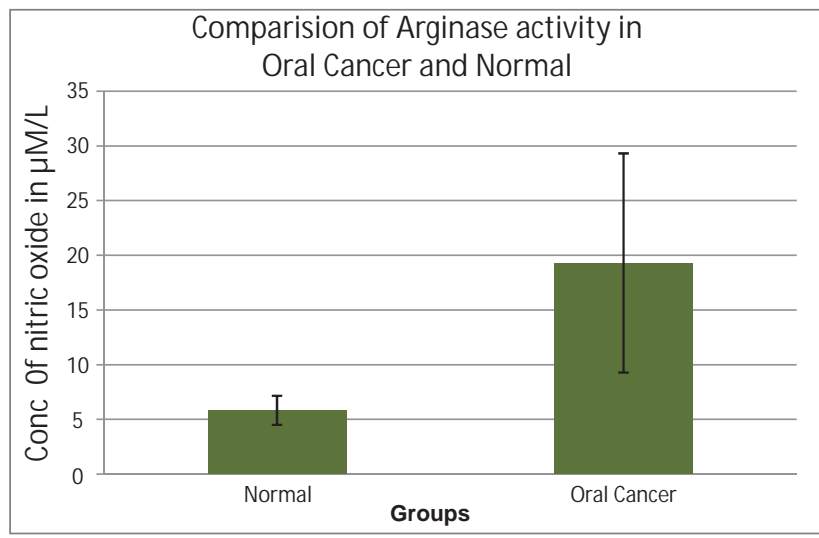




\section{References :}

1. Shou-Yen Kao, Yu-Wei Chu, Ya-Wei Chen, Kuo-Wei Chang, Tsung-Yun Liu. Detection and Screening of Oral Cancer and Pre-cancerous Lesions. J Chin M ed Assoc 2009; 72(5): 227-33.

2. Jenkin DC, Charles IG, Thomson LL, M oss DW, Holmes LS, Baylis SA, et al. Role of nitric oxide in tumor growth. Proc Natl Acad Sci USA 1995; 92:4392-6.

3. Patel RP, M CAndrew J, Sellak H, White RC, Jo H, Freeman BA, et al. Biological aspects of reactive nitrogen species. Biochim Biophys Acta 1999; 1411:385- 400.

4. Hogg N, Singh RJ, Kalyanaraman B. The role of glutathione in the transport and catabolism of nitric oxide. FEBS Lett 1996; 382:223-8.

5. Li RH, HotchkissJH. Potential genotoxicity of chronically elevated nitric oxide: a review. Mutat Res 1995; 339:73-89.

6. Beevi, SS., Rasheed, AM ., Geetha, A.Evaluation of oxidative stress and nitric oxide levels patients with oral cavity cancer.jpn J Clin 0 ncol.pages 2002: 379-385.

7. Gallo O., M asini E., M orbidelli L., Franchi A., Fini-Storchi., Vergari, W A and Ziche, M., (1998).Role of nitric oxide in angiogenesis and tumor progression in head and neck cancer. 1998; 90(24):587-596.

8. Connelly,S., Macabeo-Ong, M.,Dekker N.,Jordan R.,Schmidt B., Increased NO levels and iNOS over expression in oral squamous cell carcinoma, oral oncology, 41 (3):261-267.
9. Fukumura, D., Kashiwagi, S., Jain, RK. The role of nitric oxide in tumor progression. Nature reviews cancer. 2006;6: 521-534.

10. Park S, Lee $S$, Song $S$, Heo D, Park B, Lee $D$, et al., The Effect of Nitric Oxide on Cyclooxygenase-2 (Cox-2) Overexpression in Head and Neck Cancer Cell Lines. Int. J. Cancer: 107, 729-738.

11. Kepka-Lenhart,D.,M istry,S.K.,Wu,G and Morris Jr,M. Arginase:a limiting factor for nitric oxide and polyamine synthesis by activated macrophages. Am J Physiol regulatory integrative comp physiol 2000; 279(6): R2237-R2242.

12. Chang, Cl., Liao J.C and Kuo, L. M acrophage arginase promotes tumor cell growth and suppresses nitric oxide-mediated tumor Cytotoxicity. Cancer Res 2001; 61(3):1100-1106.

13. Peter Popovic J., Herbert Zeh J, III and Juan Ochoa B. Arginine and Immunity.J of Nutrition 2007:1681-86.

14. Bansal., Vishal Ochoa and Juan B. Arginase availability, arginase, and the immune response. Current Opinion in Clinical Nutrition and Metabolic Care 2003; 6(2):223-28.

15. Li RH, Hotchkiss JH. Potential genotoxicity of chronically elevated nitric oxide: a review. Mutat Res 1995; 339:73-89.16. Hesse,M .,M odolell,M .,LaFlamme, A.C.,Schito,M .,Fuentes, J.M .,Cheev er,A.W.,Pearce,J and Wyan,T.A. Differential regulation of nitric oxide synthase- 2 and arginase-1 by type1/type2 cytokines in vivo. J.Immunol 2001; 167(11): 6533-6544. 